General

\title{
The Effect of Nonlexical Verbal Signals on the Perceived Authenticity, Empathy and Understanding of a Listener
}

\author{
Luca M Leisten ${ }^{1} \odot$, Franziska Findling', Jonas Bellinghausen', Max Kinateder ${ }^{2}$, Thomas Probst ${ }^{3}$, Despina Lion ${ }^{4}$, Youssef \\ Shiban \\ ${ }^{1}$ Clinical Psychology and Psychotherapy Research, PFH Göttingen, ${ }^{2}$ National Research Council, Ottowa, Canada, ${ }^{3}$ Psychotherapy and \\ Biopsychosocial Health, Danube University Krems, ${ }^{4}$ Health Psychology and Sports Psychology, PFH Göttingen \\ Keywords: nonlexical verbal signals, speech perception, cbt, therapeutic relation, active listening \\ https://doi.org/10.46853/001c.27434
}

The European Journal of Counselling Psychology

Vol. 10, Issue 1, 2021

Active listening plays an important role in the relationship between clients and therapists. Here, we investigated whether variations of the confirmatory nonlexical verbal communication signal "mmh" influenced perceived authenticity, empathy, and understanding of a listener. Eighty-one participants were in a conversation with an interviewer and reported about a difficult work experience. They were randomly assigned to one of three groups: the control group did not receive any verbal feedback from the interviewer; in one experimental group ( $1 \mathrm{x}-\mathrm{mmh})$, the interviewer uttered several monosyllabic confirmatory nonlexical verbal signals ("mmh") during the presentation; in a second experimental group ( $3 \mathrm{x}-\mathrm{mmh})$ the interviewer voiced several tri-syllabic "mmh-mmh-mmh" while listening. All participants were then asked to rate the perceived authenticity, empathy, and understanding of the interviewer. Participants in the $3 \mathrm{x}-\mathrm{mmh}$ condition rated the interviewer to be significantly less authentic than those in the other two groups. No differences in reported empathy and understanding were found. The use of consecutive confirmatory nonlexical verbal signals ("mmh"), at least as currently implemented, may influence the perceived authenticity of a listener.

\section{Introduction}

Active listening is an important instrument of communication between psychotherapists and their clients (Robertson, 2005). Active listening includes various techniques for gathering information. Among them are the use of open questions, summarizing, paraphrasing or verbalization, and clarification (Simpson et al., 1991). An active listener is expected to avoid spontaneous reactions when actively listening, as these are thought to be disruptive to communication. In this way the client and his or her needs can be acknowledged and given the opportunity to express themselves actively. Consequently, the therapist's communication with the client is facilitated by active listening, not only verbally, but also nonverbally (Robertson, 2005). Active listening is generally considered to be beneficial for the client, e.g., through a pleasant and quiet environment. An environment can be created in which clients can express themselves well and without fear, feeling understood, and accepted (Martin et al., 2017).

Several studies have examined the effects of active listening and communication between therapist and client on therapy success. Nugent and Halvorson (1995), for example, showed that active listening can reduce the symptoms of depression. Wanzer and colleagues (2004) further investigated client-centered communication and its effects on client satisfaction using a survey. It was found that clientcentered communication had a positive influence on client satisfaction with treatment and communication. Martin and colleagues (2017), in turn, investigated active listening both as an attitude of the therapist and a technique to find out what influence this distinction has on the satisfaction of the therapist-client relationship. In their study, active listening was investigated with a changed mediation in therapist-client conversations. Three groups, 19 students in total, were recruited, one of which was trained in active listening as an attitude. The second group was trained with regard to technique, while the third group was a control group. The comparison of the two intervention groups showed that the group that received attitude-oriented training responded significantly better to clients' feelings than the technique-oriented group. This reflects that active listening can express empathy and understanding.

Nonverbal communication in general can promote trusting relationships. A systematic review by Lorié and colleagues (2017) investigated how different cultures transmit nonverbal expressions of empathy and what effects this has on therapist-client relationships. Empathy is described as the ability to perceive verbal and nonverbal emotional cues of a counterpart and to react to them. Empathy can be expressed not only through verbal but also through purely nonverbal behavior (Riess \& Kraft-Todd, 2014). The review concluded that the quality of communication is influenced by nonverbal expressions of empathy, which in turn influenced client satisfaction.

There are several ways in which a therapist can implement active listening; in turn, clients may differ in how they perceive certain active listening techniques. Investigating 
how active listening influences the perception of the therapist has important implications for counselling therapy as it is possibly associated with client satisfaction. Ultimately, whether a client perceives their therapist as empathic, understanding, and authentic (i.e., expressing oneself according to one's own true emotions, needs, thoughts, and beliefs; Wirtz et al., 2020) can influence therapy success.

Active listening can be signaled through different channels, one of them being the use of affirmative nonlexical verbal signals such as "mmh" or "aha", which serve as empathic continuers to show emotional involvement and understanding (Fitzgerald \& Leudar, 2010). Similarly, empathic continuers serve as a "back-channel response" to keep the conversation going and signal attention to the client (Bodie et al., 2012). In addition, there are nonverbal signals of active listening, such as a simple nod, an open posture or eye contact. Pinto et al. (2012), for example, found a positive correlation between such interaction styles during a therapeutic session and the reported empathy of the therapists.

Other studies on verbal and nonverbal signals were less conclusive. Henry and colleagues (2012) investigated the effects of nonverbal communication between clients and therapists during clinical interactions in a meta-analysis. They concluded that both warmth and perceived listening on the therapist's side had a positive impact on client satisfaction. However, the authors emphasized that further studies are needed to investigate the influence of nonverbal signals on client satisfaction (Henry et al., 2012). What remains unclear is how the use of different types of empathic continuers influences the relationship between therapist and client.

To fill in this research gap and extend the work of Martin et al. (2017) and Henry et al. (2012), the present study investigated the influence of the confirmatory nonlexical verbal signal "mmh" on the perceived authenticity, empathy, and understanding of a listener in a conversation (e.g., a therapist listening to a client). We expected that variations in the nonlexical verbal confirmation signal "mmh" expressed during a conversation would increase the perceived authenticity, understanding, and empathy of the listener. Based on the results of Martin et al. (2017), which documented some initial indications of possible effects of active listening on self-reported and observed satisfaction with an interaction, we developed the following hypotheses:

$\mathrm{H} 1$ : In the $1 \mathrm{x}-\mathrm{mmh}$ condition, participants perceive interviewers to be more authentic, empathic, and understanding compared to the interviewers in the $3 \mathrm{x}-\mathrm{mmh}$ condition.

H2: In the control group (0x-mmh condition), interviewers are perceived to be more authentic, empathic, and understanding than in the $3 \mathrm{x}-\mathrm{mmh}$ condition.

H3: In the $1 \mathrm{x}-\mathrm{mmh}$ condition, interviewers are perceived to be more authentic, empathic, and understanding than interviewers in the control group.

\section{Method}

\section{Sample}

A power analysis using g*power (Faul et al., 2007) indi- cated a required sample size of $N=90$ participants to reliably detect a medium sized effect $(d=0.4$, power $=0.8)$. Our research team originally consisted of 10 undergraduate interviewers, who each collected data from nine participants, however data from one interviewer was lost in the process. Therefore, the remaining sample consisted of 81 participants (38 female and 43 male). Participants were recruited via handouts and social media in various German cities. Their average age was $M=30.28$ years $(S D=12.07$, range = 18-64). Exclusion criteria for participation were undergoing current medical or psychotherapeutic treatment or having any type of physiological condition that might have influenced participants' ability to listen, talk or see.

\section{Procedure}

The study took place at nine different locations across Germany, with nine different interviewers. All interviewers were part of a research group of the author's institution consisting of eight females and one male who were undergraduate students $\left(M_{\text {age }}=20.50, S D=1.58\right)$. Specific communication training was not provided because interaction with participants was strictly prescribed by the study protocol. The rooms in each location were chosen based on prior defined characteristics, such as being an almost plain, private room with minimal distractions and no third-party listeners. This was done to minimize the risk of any geographic effect on the results. Additionally, all nine interviewers set up the room in a similar manner, minimizing experimental noise that may have resulted from the different locations. Data collection through different interviewers carries the risk of an experimenter effect. Nevertheless, this approach was chosen to increase the validity of the results. To minimize the influence of personal characteristics of an individual interviewer, each interviewer collected data in each condition. Prior to the experiment, participants were greeted and introduced to the experiment, including information about data protection and the possibility to withdraw participation at any time.

After reading the instructions and giving written informed consent, participants were asked to report on a difficult experience at work for five minutes. In the control group the interviewer listened in silence. In the $1 \mathrm{x}-\mathrm{mmh}$ group, the interviewer voiced several monosyllabic confirmatory nonlexical verbal signals (" $\mathrm{mmh}$ ") while listening. In the $3 x-m m h$ group the interviewer repeatedly said "mmh-mmh-mmh" while listening. After the report, participants completed a written questionnaire that captured the dependent variables, perceived authenticity, empathy, and understanding. Finally, participants were debriefed about the purpose of the experiment and had the opportunity to ask questions.

Participants were randomly assigned to the three groups, with each group consisting of $n=27$ participants. The participants were assigned to an interviewer based on geographic proximity to the closest test location. The participants were randomized by the order of their appointments with the interviewer; the first participant was placed in the control group, the second in the $1 \mathrm{x}-\mathrm{mmh}$ condition, the third in the $3 \mathrm{x}-\mathrm{mmh}$ condition, and so on. Thus, each interviewer performed all conditions in a fixed order. During 
the participant's verbal report the interviewer either said nothing (control group), or uttered the nonlexical signals several times "mmh" (1x-mmh condition) or "mmh-mmhmmh" (3x-mmh condition). The interviewer attempted to interject the "mmh" whenever it appeared appropriate during the participant's report (e.g., when there was a natural pause in the flow of the report). On average the nonlexical verbal communication signals were used roughly every 60 seconds (about five times during each verbal report). The exact number of the interjections was not recorded. In order to eliminate other nonverbal interferences, the behavior of the interviewer was strictly prescribed by the study protocol (e.g., body posture upright, neutral voice during greeting, eye contact during the experiment).

\section{Measures}

For the purpose of this study, we developed a new questionnaire based on previous work. Existing inventories measuring perceived authenticity, empathy, and understanding either include too many items regarding a personal relationship between the participant and the interviewer (Ganley, 1989; in our study, participants did not know the interviewers), have poor content and construct validity (Holz-Ebeling \& Steinmetz, 1995), or are self-reporting measures (Kernis \& Goldman, 2006; van den Bosch $\&$ Taris, 2014). The definitions (Wirtz et al., 2020) of the dependent variables were deductively operationalized to items, and existing questionnaires were used for additional guidance (Authenticity: Kernis \& Goldman, 2006; van den Bosch \& Taris, 2014; Empathy: Barrett-Lennard, 1962; Kim et al., 2004; Understanding: Barrett-Lennard, 1962). As the focus of our study was to investigate the perception of a listener with no prior relationship, only items that could be translated to perceptions of others were chosen and rephrased for our purposes.

The resulting questionnaire, called the socio-emotional perception scale (SEPS-I), measured the perceived authenticity (ten items), empathy (eight items), and understanding (five items; see supplementary material for an English version of the questionnaire, translated from German). Questions measuring the perceived authenticity included items such as "I think my listener was showing his/her real emotions", while empathy was measured using items similar to "I think my listener put him-/herself in my shoes". To measure understanding, the questionnaire consisted of items like "I think the listener understood my concerns". In addition, social desirability was captured by items like "I never stole anything in my life".

Each item was rated on a Likert scale ranging from $1=$ fully disagree to 6 = fully agree. A score for perceived authenticity, empathy, and understanding was calculated by taking the average of the items for each construct. Higher values on each scale correspond to higher perceived authenticity, empathy, and understanding of the listener. For each scale, Cronbach's alpha was calculated, yielding $\alpha=.90$ for authenticity, $\alpha=.88$ for empathy, and $\alpha=.81$ for understanding, showing an overall high reliability.

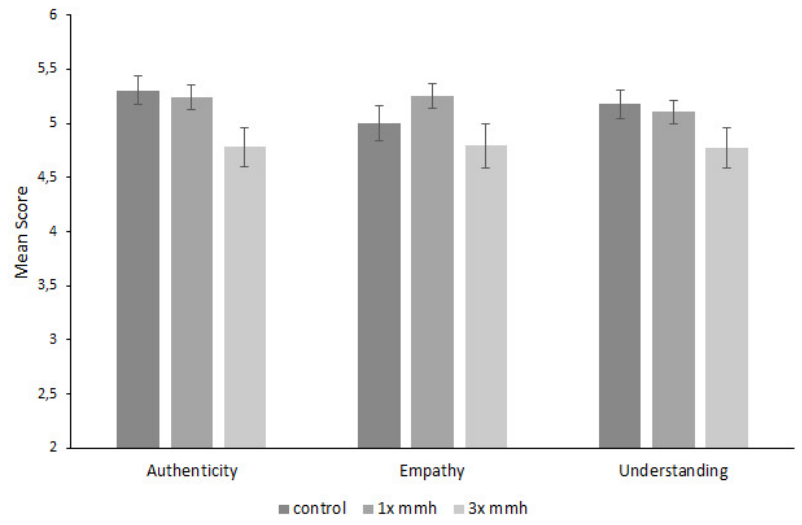

Figure 1. Means of perceived authenticity, empathy and understanding per condition

Note. Error bars represent SEM. The $\mathrm{x}$-axis starts at two.

\section{Statistical Analysis}

IBM SPSS Statistics 22 (IBM Corp; Armonk, NY) was used for statistical analysis. Univariate ANOVAs were used to test for significant differences. The procedure was chosen even though the data was not normally distributed, given its robustness against violation of this assumption. Least Significant Difference (LSD) tests were used for follow-up testing and the Bonferroni correction was applied where necessary. The significance level was set to .05 a priori.

\section{Results}

The demographic variables age (Kruskal-Wallis test, $p=$ $.09)$ as well as gender $(p=.95)$ did not significantly differ between conditions. No other demographic variables were examined. Figure 1 illustrates the mean scores of the three dependent variables by group.

A univariate ANOVA was performed to test for significant differences between conditions. There was a significant main effect of condition on authenticity, $F(2,78)=3.65, p=$ .03, $\omega=0.06$, but not on empathy, $F(2,78)=1.97, p=.15$, $\omega=0.02$, nor on understanding, $F(2,78)=2.13, p=.13$, $\omega$ $=0.03$. Post-hoc tests were performed to further investigate the condition differences in perceived authenticity. An LSDtest showed significant differences in perceived authenticity between the control group and the $3 \mathrm{x}-\mathrm{mmh}$ condition, $t(78)=-2.476, p=.02\left(p_{\text {bonferroni }}=.046\right), d=0.78$, indicating that authenticity was higher in the control group $(M=$ $5.30, S E=0.13)$ than in the $3 \mathrm{x}-\mathrm{mmh}$ condition $(M=4.78, S E$ $=0.19$ ). After correcting for multiple comparisons (Bonferroni), no significant differences in authenticity were found between the control group $(M=5.30, S E=0.13)$ and the $1 \mathrm{x}$ mmh condition $(M=5.24, S E=0.12), t(78)=-0.30, p=.77$ ( $\left.p_{\text {bonferroni }}=1\right), d=0.10$, nor between the $1 \mathrm{x}-\mathrm{mmh}$ condition and the $3 x-m m h$ condition, $t(78)=-2.178, p=.03$ ( $p_{\text {bonferroni }}$ $=.10), d=0.47$. Consequently, the data supported hypothesis 2 , but hypotheses 1 and 3 had to be rejected.

\section{Discussion}

The objective of the present study was to investigate the 
effect of nonlexical verbal signals on the perceived authenticity, empathy, and understanding of a listener. In summary, the results show that the extensive use of confirmatory nonlexical verbal signals may decrease perceived authenticity of a listener in some cases. The perceived authenticity was lower if one used three consecutive "mmh" compared to no verbal signal, but there was no difference between three "mmh" and one "mmh" after correcting for multiple testing. Contrary to our expectations and previous work (Martin et al., 2017), we did not find evidence that the verbal signals used in conversations influenced perceived empathy or understanding. Our second hypothesis, stating that the control group perceived the interviewer as more authentic, empathetic, and understanding than the $3 \mathrm{x}-$ mmh condition, was partially supported, as only perceived authenticity was influenced by the manipulation. Our first and third hypotheses were rejected, as there were no differences between conditions.

There are several possible explanations for the results observed in the present study. For instance, we speculate that nonverbal communication through body language might have gained more importance in the control group where there was no verbal communication. Therefore, the control group might have been particularly influenced by cues like facial expressions or posture. This, however, needs to be tested in a future study. Additionally, it is possible that the $3 \mathrm{x}-\mathrm{mmh}$ condition appeared artificial to participants, compared to no or monosyllabic continuers, resulting in reduced perceived authenticity.

Contrary to earlier work (Martin et al., 2017), we did not find an influence of verbal signals on the empathy or understanding of a listener, but we did find an effect on the perceived authenticity. These findings can serve as first insights into the importance of nonlexical verbal signals on the perceived authenticity of a listener, which in turn could possibly influence client satisfaction in therapy. Our study extends the findings by Martin et al. (2017) and Henry et al. (2012), who only showed an influence of active listening on understanding and patient satisfaction and confirms the importance of active listening signals in conversations. Additionally, previous research (Bodie et al., 2012) indicated that verbal signals can be associated with friendliness and responsiveness. Our results expand this work, showing that some forms of nonlexical verbal signals may be less effective than others in communicating the authenticity of a listener. Although future research is clearly needed to address the inconsistencies in results, practitioners using active listening need to be aware that not all techniques may be equally effective.

\section{Limitations and Recommendations for Future Research}

There are several limitations to the present study. First and foremost, the generalization of the results is limited. Due to the online recruitment of participants, the representativeness of the sample is not guaranteed, which is a common limitation in our field. Second, the questionnaire is not tested regarding its reliability and validity. Even though Cronbach's alpha was high for the developed questionnaire, future work is needed to compare the present tool to an es- tablished psychometric survey instrument (e.g., Tscheulin \& Splitter, 2001). Third, since there is no documentation of the exact timing and number of interjections in the $1 \mathrm{x}$ $\mathrm{mmh}$ and $3 \mathrm{x}-\mathrm{mmh}$ conditions, as well as the nonverbal behavior, it is difficult to gauge which aspects of the manipulation are responsible for the observed results. Fourth, the different locations and interviewers used may have resulted in slight experimental noise and an experimenter effect, although the best possible has been done to minimize those effects through the actions described above. Fifth, the initial (welcome and consent) and final interactions between participant and interviewer may have been remembered particularly vividly but were not part of the experiment itself (Primacy or Recency-Effect; Atkinson \& Shiffrin, 1986). Finally, no objective measurements (e.g., videotaping) of the interviews were taken. It cannot be ruled out that the interviewers inadvertently expressed verbal or other behavior during the consenting phase and even the experiment that may have also influenced the perception. Future iterations of this study should include two experimenters, one responsible for instruction, study procedure, and assessment of the interviewer's emitted nonverbal communication, and another taking on the role of the interviewer.

Practical limitations that need to be accounted for include the nonclinical experimental setting. The study scenario and sample, although attempting to mimic a clinical setting, differed from most clinical settings. This limits the generalizability of the present findings to clinical work, as the results ideally would need to be tested again, for example with clients in a psychotherapeutic setting.

In general, most participants across the control group and the two experimental conditions reported relatively high authenticity, empathy, and understanding scores. Future research should investigate situations in which participants would be expected to trust listeners less (e.g., between a suspect in a criminal investigation and the police) to test if active listening techniques can increase authenticity, empathy, or understanding in more challenging settings. Additionally, it would be interesting to investigate whether the effect of verbal signals on authenticity can be replicated in a clinical setting as this would make the results more generalizable. As emphasized above, body language and facial cues could be manipulated, in order to test whether some variability in the perceived authenticity, understanding, and empathy can be accounted for by either of these. Finally, as authenticity was the only variable influenced by the verbal signal's manipulation, it would be interesting to focus on how authenticity is influenced by other verbal signals (e.g., "aha”) or signal properties (e.g., intonation; lexical vs. nonlexical signals), and how these can be used to increase the perceived authenticity in order to improve client satisfaction.

\section{Implications for Practice}

Our findings have important implications for Counseling Psychology in Europe. We showed that nonlexical verbal signals might influence the perception of a listener, indicating a possible impact on the relationship between client and therapist. Ultimately, our research can inform the European counseling community about the tools available to 
strengthen therapist-client relationships.

\section{Summary and Conclusion}

The present study investigated the effect of nonlexical verbal signals on the perceived authenticity, empathy, and understanding of a listener. The results showed that the extensive use of confirmatory nonlexical verbal signals may decrease perceived authenticity of a listener in some cases. We did not find evidence that the verbal signals used in con- versations influenced perceived empathy or understanding. Future research is needed to confirm and further explore our results, but practitioners using active listening need to be aware that not all techniques may be equally effective.

Submitted: October 05, 2020 EET, Accepted: August 13, 2021

EET 


\section{REFERENCES}

Atkinson, R. C., \& Shiffrin, R. M. (1986). Human Memory: A proposed system and its control processes. In K. W. Spence \& J. T. Spence (Eds.), The psychology of learning and motivation (2nd ed., pp. 89-195). Academic Press. https://doi.org/10.1016/s00 79-7421(08)60422-3

Barrett-Lennard, G. T. (1962). Dimensions of therapist response as causal factors in therapeutic change. Psychological Monographs: General and Applied, 76(43), 1-36. https://doi.org/10.1037/h0093918

Bodie, G. D., Cyr, K., Pence, M., Rold, M., \& Honeycutt, J. (2012). Listening Competence in Initial Interactions I: Distinguishing Between What Listening Is and What Listeners Do. https://doi.org/10.1080/10904018.2012.6 $\underline{39645}$

Faul, F., Erdfelder, E., Lang, A.-G., \& Buchner, A. (2007). $G^{*}$ Power 3: A flexible statistical power analysis program for the social, behavioral, and biomedical sciences. Behavior Research Methods, 39(2), 175-191. https://doi.org/10.3758/bf03193146

Fitzgerald, P., \& Leudar, I. (2010). On active listening in person-centred, solution-focused psychotherapy. School of Psychological Sciences. https://doi.org/10.1016/i.pr agma.2010.07.007

Ganley, R. M. (1989). The Barrett-Lennard Relationship Inventory (BLRI): Current and potential uses with family systems. Family Process, 28(1), 107-115. http s://doi.org/10.1111/j.1545-5300.1989.00107.x

Henry, S. G., Fuhrel-Forbis, A., Rogers, M. A. M., \& Eggly, S. (2012). Association between nonverbal communication during clinical interactions and outcomes: A systematic review and meta-analysis. Patient Education and Counseling, 86(3), 297-315. http s://doi.org/10.1016/j.pec.2011.07.006

Holz-Ebeling, F., \& Steinmetz, M. (1995). Wie brauchbar sind die vorliegenden Fragebogen zur Messung von Empathie? Kritische Analysen unter Berücksichtigung der Iteminhalte. Zeitschrift für Differentielle und Diagnostische Psychologie, 16, 1132.

Kernis, M. H., \& Goldman, B. M. (2006). A multicomponent conceptualization of authenticity: Theory and research. In M. P. Zanna (Ed.), Advances in experimental social psychology (Vol. 38, pp. 283-357). Elsevier Academic Press. https://doi.org/10.1016/s006 $\underline{\text { 5-2601(06)38006-9 }}$

Kim, S. S., Kaplowitz, S., \& Johnston, M. V. (2004). The effects of physician empathy on patient satisfaction and compliance. Evaluation \& the Health Professions, 27(3), 237-251. https://doi.org/10.1177/01632787042 67037
Lorié, Á., Reinero, D. A., Phillips, M., Zhang, L., \& Riess, H. (2017). Culture and nonverbal expressions of empathy in clinical settings: A systematic review. Patient Education and Counseling, 100(3), 411-424. htt ps://doi.org/10.1016/j.pec.2016.09.018

Martin, O., Rockenbauch, K., Kleinert, E., \& StöbelRichter, Y. (2017, September). Aktives Zuhören effektiv vermitteln: Zwei Konzepte im Vergleich. Der Nervenarzt, 1026-1035. https://doi.org/10.1007/s0011 5-016-0178-X

Nugent, W. R., \& Halvorson, H. (1995). Testing the effects of active listening. Research on Social Work Practice, 5(2), 152-175. https://doi.org/10.1177/10497 3159500500202

Pinto, R. Z., Ferreira, M. L., Oliveira, V. C., Franco, M. R., Adams, R., Maher, C. G., \& Ferreira, P. H. (2012). Patient-centred communication is associated with positive therapeutic alliance: A systematic review. Journal of Physiotherapy, 58(2), 77-87. https://doi.org/ 10.1016/s1836-9553(12)70087-5

Riess, H., \& Kraft-Todd, G. (2014). E.M.P.A.T.H.Y.: A Tool to Enhance Nonverbal Communication Between Clinicians and Their Patients. Academic Medicine, 89(8), 1108-1112. https://doi.org/10.1097/acm.00000 00000000287

Robertson, K. (2005). Active listening: More than just paying attention. Australian Family Physician, 34(12), $1053-1055$

Simpson, M., Buckman, R., Stewart, M., Maguire, P., Lipkin, M., Novack, D., \& Till, J. (1991). Doctorpatient communication: The Toronto consensus statement. BMJ, 303(6814), 1385-1387. https://doi.or $\mathrm{g} / 10.1136 / \mathrm{bmj} .303 .6814 .1385$

Tscheulin, D., \& Splitter, S. (2001). Zur Messung von erlebtem Beziehungsverhalten. Kurzbericht über ein Inventar für Beziehungsverhalten (IBV). In I. Langer (Ed.), Menschlichkeit und Wissenschaft. Festschrift zum 80. Geburtstag von Reinhard Tausch (pp. 363-372). GwG-Verlag.

van den Bosch, R., \& Taris, T. W. (2014). Authenticity at work: Development and validation of an individual authenticity measure at work. Journal of Happiness Studies, 15(1), 1-18. https://doi.org/10.1007/s10902-0 13-9413-3

Wanzer, M. B., Booth-Butterfield, M., \& Gruber, K. (2004). Perceptions of Health Care Providers' Communication: Relationships Between PatientCentered Communication and Satisfaction. Health Communication, 16(3), 363-384. https://doi.org/10.12 07/s15327027hc1603_6

Wirtz, M. A., Dorsch, F., \& Verlag Hans Huber. (2020). Dorsch - Lexikon der Psychologie. Hogrefe. https://do i.org/10.1024/85914-000 


\section{SUPPLEMENTARY MATERIALS}

\section{Supplementary Material}

Download: https://ejcop.scholasticahq.com/article/27434-the-effect-of-nonlexical-verbal-signals-on-the-perceivedauthenticity-empathy-and-understanding-of-a-listener/attachment/ 69004.docx?auth token=Y91H3U139FpH3js07SpS 